\title{
Probing the chiral limit with clover fermions II: The baryon sector
}

QCDSF/UKQCD Collaboration: Meinulf Göckeler ${ }^{a}$, Philipp Hägler ${ }^{b}$, Roger Horsley ${ }^{c}$, Yoshifumi Nakamura $^{d}$, Munehisa Ohtani $^{a}$, Dirk Pleiter ${ }^{* d}$, Paul E.L. Rakow ${ }^{e}$, Andreas Schäfer $^{a}$, Gerrit Schierholz $^{d f}$, Wolfram Schroers ${ }^{d}$, Hinnerk Stüben ${ }^{g}$ and James

M. Zanotti ${ }^{c}$

${ }^{a}$ Institut für Theoretische Physik, Universität Regensburg, 93040 Regensburg, Germany

${ }^{b}$ Institut für Theoretische Physik T39, Physik-Department der TU München, 85747 Garching, Germany

${ }^{c}$ School of Physics, University of Edinburgh, Edinburgh EH9 3JZ, UK

${ }^{d}$ John von Neumann-Institut für Computing NIC, Deutsches Elektronen-Synchrotron DESY,

15738 Zeuthen, Germany

e Theoretical Physics Division, Department of Mathematical Sciences, University of Liverpool, Liverpool L69 3BX, UK

${ }^{f}$ Deutsches Elektronen-Synchrotron DESY, 22603 Hamburg, Germany

${ }^{g}$ Konrad-Zuse-Zentrum für Informationstechnik Berlin, 14195 Berlin, Germany

Email: dirk.pleiter@desy.de

\begin{abstract}
Algorithmic progress in recent years made it possible to simulate QCD with $N_{f}=2$ flavours of $O(a)$-improved Wilson fermions at very light quark masses. We present the current results for baryon spectrum states, the nucleon axial coupling and the lowest moment of unpolarised nucleon structure functions. Special emphasis is given to a comparison of our calculations with results from chiral effective theories.
\end{abstract}

The XXV International Symposium on Lattice Field Theory

July 30 - August 42007

Regensburg, Germany

\footnotetext{
* Speaker.
} 


\section{Introduction}

Thanks to recent algorithmic improvements and the availability of a new generation of capability computers, simulations with dynamical Wilson fermions can now be extended to much lighter quark masses and bigger lattices. In recent years the QCDSF collaboration has significantly extended the number of ensembles of gauge configurations with $N_{f}=2$ flavours of $O(a)$-improved Wilson fermions. These datasets cover a large range of quark masses as well as different lattice spacings and volumes. In principle, this puts us in the position to check for systematic errors that affect essentially all lattice calculations, i.e. finite size corrections, discretisation errors and errors due to extrapolations to the physical quark masses or the chiral limit.

For the analysis of both, finite size effects and the quark mass dependence, results from chiral effective theories can be used to correct the simulation results or to guide extrapolations. Although the strategy of combining the results obtained from lattice simulations and chiral effective theories has led to a consistent picture for various observables, e.g. the pion decay constant [1], the mass of the nucleon [2], the axial coupling of the nucleon [3] or the nucleon electromagnetic form factors [4], this approach suffers from the paucity of lattice results in the region where chiral perturbation theory is expected to be applicable.

Another source for systematic errors stems from the translation of the lattice results into physical units. A quantity which is often used for this purpose is the Sommer scale $r_{0}$. While this quantity has the advantage that on the lattice it can be determined with relatively high statistical accuracy, its experimental value is far less well known. To avoid depending on this experimental value, which relies on model assumptions, one can alternatively request lattice results for a quantity $X$, which has the dimension of a mass (e.g. the pion decay constant or the mass of the nucleon), to be equal to the corresponding experimental result, i.e. $r_{0}^{\text {lat }} X^{\text {lat }}=r_{0} X^{\text {exp }}$. The results from different collaborations suggest that the resulting value for $r_{0}$ is significantly smaller than the typically quoted experimental number $r_{0} \simeq 0.5 \mathrm{fm}$. In this paper we will use $r_{0}=0.467 \mathrm{fm}$ which allows
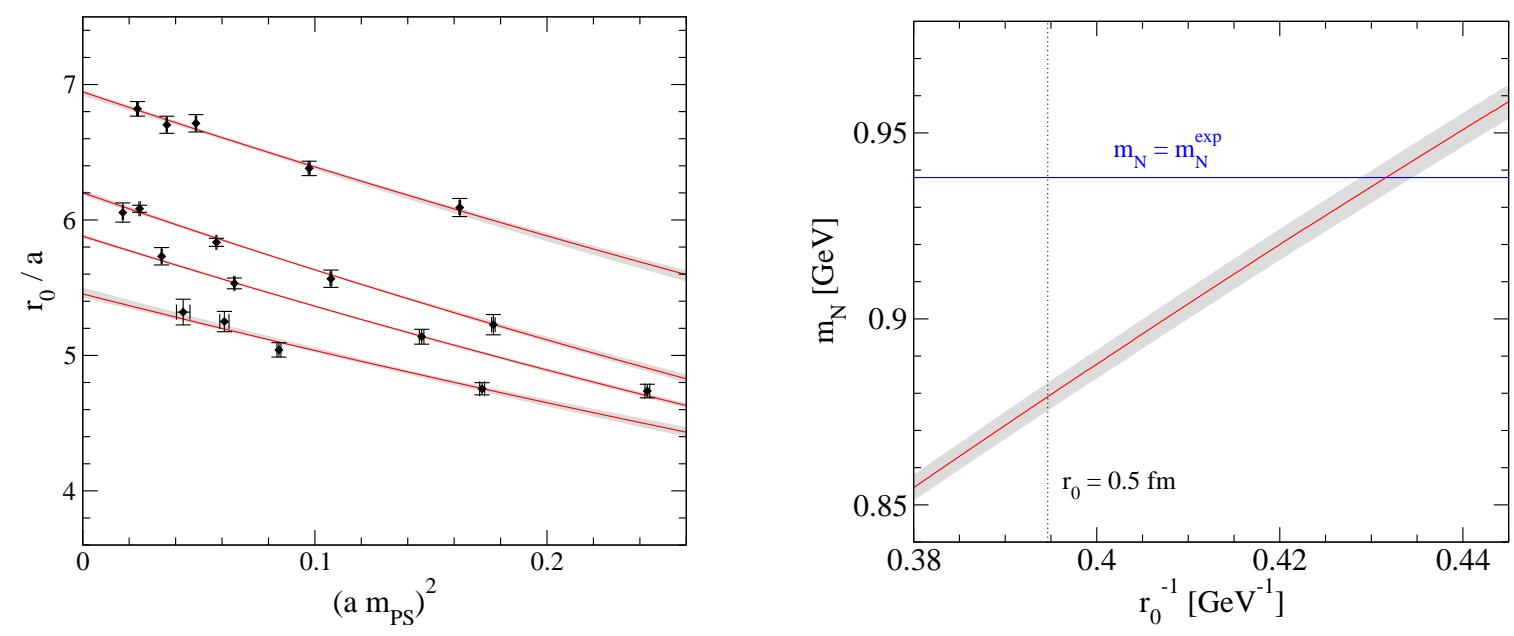

Figure 1: The left plot shows the values for $r_{0}\left(\beta, \kappa^{\mathrm{S}}\right)$ together with a fit to Eq. (1.1). In the right plot $m_{\mathrm{N}}\left(m_{\mathrm{PS}}=m_{\pi}\right)$, which is determined from fits to Eq. (2.1) for different values of $r_{0}$, is shown as a function of $r_{0}^{-1}$. 
for easy comparison with our previous results. Our most recent results for the nucleon mass (see next section and Fig. 1) and the pion decay constant (see [1]) suggest that the actual value is even smaller. To define our scale independent of the quark mass we extrapolate the measured values of $r_{0}^{\text {lat }}\left(\beta, \kappa^{\mathrm{S}}\right)$ to the chiral limit using the ansatz

$$
\ln \frac{r_{0}^{\text {lat, }}}{a}=A_{0}(\beta)+A_{2}(\beta)\left(\operatorname{am}_{\mathrm{PS}}\left(\beta, \kappa^{\mathrm{S}}\right)\right)^{2},
$$

where $A_{i}(\beta)=A_{i 0}+A_{i 1}\left(\beta-\beta_{0}\right)+A_{i 2}\left(\beta-\beta_{0}\right)^{2}$ and $\beta_{0}=5.29$. We fit our data in the range am $_{\mathrm{PS}}<$ 0.5 and find $\chi^{2} / N_{\text {d.o.f. }}=6.7 / 12$ (see Fig. 1 ).

\section{Masses}

The mass of the nucleon, $m_{\mathrm{N}}$, and delta, $m_{\Delta}$, are experimentally well-determined quantities. However, for both quantities calculations using baryon chiral perturbation theory (B $\chi \mathrm{PT}$ ) suggest a rather non-trivial quark mass dependence. In an infinite volume $m_{\mathrm{N}}$ depends on the quark mass in the following way [2]:

$$
\begin{aligned}
m_{\mathrm{N}}\left(m_{\mathrm{PS}}\right)= & M_{0}-4 c_{1} m_{\mathrm{PS}}^{2}-\frac{3 g_{A, 0}^{2}}{32 \pi F_{0}^{2}} m_{\mathrm{PS}}^{3}+ \\
& {\left[e_{1}^{r}(\lambda)-\frac{3}{64 \pi^{2} F_{0}^{2}}\left(\frac{g_{A, 0}^{2}}{M_{0}}-\frac{c_{2}}{2}\right)-\frac{3}{32 \pi^{2} F_{0}^{2}}\left(\frac{g_{A, 0}^{2}}{M_{0}}-8 c_{1}+c_{2}+4 c_{3}\right) \ln \frac{m_{\mathrm{PS}}}{\lambda}\right] m_{\mathrm{PS}}^{4} } \\
& +\frac{3 g_{A, 0}^{2}}{256 \pi F_{0}^{2} M_{0}^{2}} m_{\mathrm{PS}}^{5}+O\left(m_{\mathrm{PS}}^{6}\right) .
\end{aligned}
$$

Even extending our fit interval to $0<m_{\mathrm{PS}} \lesssim 650 \mathrm{MeV}$ does not allow us to determine all parameters. We therefore restrict the set of free fit parameters to the nucleon mass in the chiral limit $M_{0}$, the not very well known low-energy constant (LEC) $c_{1}$ and the counter-term $e_{1}^{r}(\lambda)$ (we use $\lambda=1 \mathrm{GeV}$ ). For the other parameters, i.e. the LECs $c_{2}$ and $c_{3}$, the pion decay constant $F_{0}$ and the nucleon axial coupling $g_{A, 0}$, we use the phenomenological estimates listed in Table 1. Our nucleon mass data and the fit are shown in Fig. 2. Note that all our results seem to fall on a universal curve, indicating discretisation effects to be small. We therefore ignored $O\left(a^{2}\right)$ effects in our fit ansatz. We observe that $m_{\mathrm{N}}\left(m_{\mathrm{PS}}=m_{\pi}\right)$ is consistent with experiment. Furthermore, we find $c_{1}=-1.02(7) \mathrm{GeV}^{-1}$, a value which is consistent with other estimates [11]. We have repeated our analysis for different values for $r_{0}$. The results are shown in Fig. 1. If we set $m_{\mathrm{N}}=m_{\mathrm{N}}^{\exp }$ we obtain $r_{0}=0.457(3) \mathrm{fm}$.

To extrapolate our results for the mass of the delta $m_{\Delta}\left(m_{\mathrm{PS}}\right)$ we fit these to the second order small scale expansion (SSE) expression [10]

$$
m_{\Delta}\left(m_{\mathrm{PS}}\right)=M_{\Delta, 0}-4 a_{1} m_{\mathrm{PS}}^{2}-\frac{3}{32 \pi F_{0}^{2}} \frac{25 h_{A}^{2}}{81} m_{\mathrm{PS}}^{3},
$$

\begin{tabular}{|l|l|l||l|l|l||l|l|l|}
\hline$c_{2}$ & $3.2 \mathrm{GeV}^{-1}$ & See [2] & $\Delta_{0}$ & $0.271 \mathrm{GeV}$ & {$[6]$} & $g_{A, 0}$ & $1.2(1)$ & {$[9]$} \\
$c_{3}$ & $-3.4 \mathrm{GeV}^{-1}$ & See [2] & $F_{0}$ & $0.0862 \mathrm{GeV}$ & {$[7]$} & $\langle\Delta x\rangle^{(u-d)}$ & 0.21 & {$[8]$} \\
$c_{A}$ & 1.5 & {$[5]$} & $M_{0}$ & $0.889 \mathrm{GeV}$ & {$[8]$} & & & \\
\hline
\end{tabular}

Table 1: Phenomenological values used as input to our fits. 

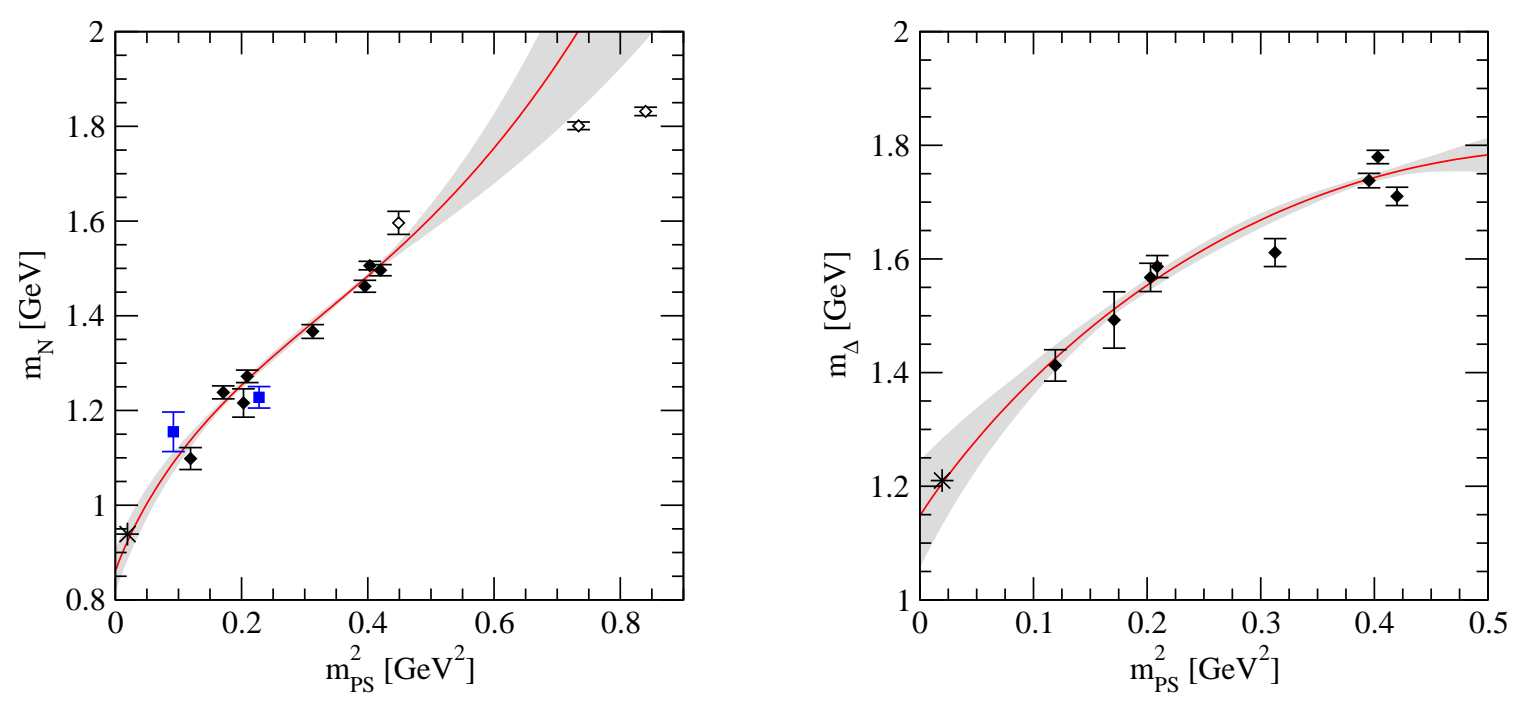

Figure 2: Lattice results for $m_{\mathrm{N}}$ (left) and $m_{\Delta}$ (right) together with a fit to Eq. (2.1) and Eq. (2.2). The open symbols indicate data which has not been included in the fit. The filled squares symbols in the left plot show preliminary data on larger lattices which has not been included in this analysis.

where we fixed $F_{0}$ to the value given in Table 1 . We find that $m_{\Delta}\left(m_{\mathrm{PS}}=m_{\pi}\right)$ is equal to the experimental value within statistical errors. The other fit parameters are consistent with expectations [10]: $a_{1}=-0.8(3) \mathrm{GeV}^{-1} \simeq c_{1}$ and $h_{A}=1.5(4) \lesssim 9 g_{A} / 5$.

\section{Nucleon axial coupling}

We will now consider the form factor of the nucleon axial current $G_{A}\left(Q^{2}\right)$ at zero momentum. ${ }^{1}$ The axial coupling constant $g_{A}=G_{A}(0)$ is determined from the renormalised axial vector current $A_{\mu}^{R}=Z_{A}\left(1+b_{A} a m_{q}\right) A_{\mu}$, where $a m_{q}=\left(1 / \kappa-1 / \kappa_{c}^{\mathrm{S}}\right) / 2$. Here we only consider the iso-vector case where contributions from disconnected terms cancel. While $Z_{A}$ is known non-perturbatively [3], $b_{A}$ is only known perturbatively and is computed using tadpole improved one-loop perturbation theory. Note that for forward matrix elements there is no need for improvement of $A_{\mu}$.

The quark mass dependence of the iso-vector nucleon axial coupling $g_{A}\left(m_{\mathrm{PS}}\right)$ has been calculated using Heavy Baryon $\chi \mathrm{PT}(\mathrm{HB} \chi \mathrm{PT})$ [3]. These calculations have been performed both in the infinite volume limit as well as for a finite spatial cubic box of length $L$. We define $g_{A}\left(m_{\mathrm{PS}}, L\right)=$ $g_{A}\left(m_{\mathrm{PS}}\right)+\Delta g_{A}\left(m_{\mathrm{PS}}, L\right)$, where $\Delta g_{A}\left(m_{\mathrm{PS}}, L\right)$ denotes the finite size effects.

We fit our data to the results obtained in [3] fixing the values for $F_{0}$, the leading axial $N \Delta$ coupling $c_{A}$ and the $N \Delta$ mass splitting at the physical point $\Delta_{0}$ to the phenomenological values given in Table 1. We furthermore set $B_{20}^{r}(\lambda)^{S S E}=0$ and use $\lambda=m_{\pi}$. A comparison of our data and the resulting fit is shown in Fig. 3.

The raw lattice data is significantly smaller than the experimental value. The fit to the $\mathrm{HB} \chi \mathrm{PT}$ expression suggests that this might be due to significant finite size effects and a rather strong quark mass dependence in the range $0<m_{\mathrm{PS}} \lesssim 300 \mathrm{MeV} . g_{A}\left(m_{\mathrm{PS}}=m_{\pi}\right)$ is found to be smaller than the

\footnotetext{
${ }^{1}$ Results for non-zero momentum have been presented by W. Schroers at this conference [4].
} 


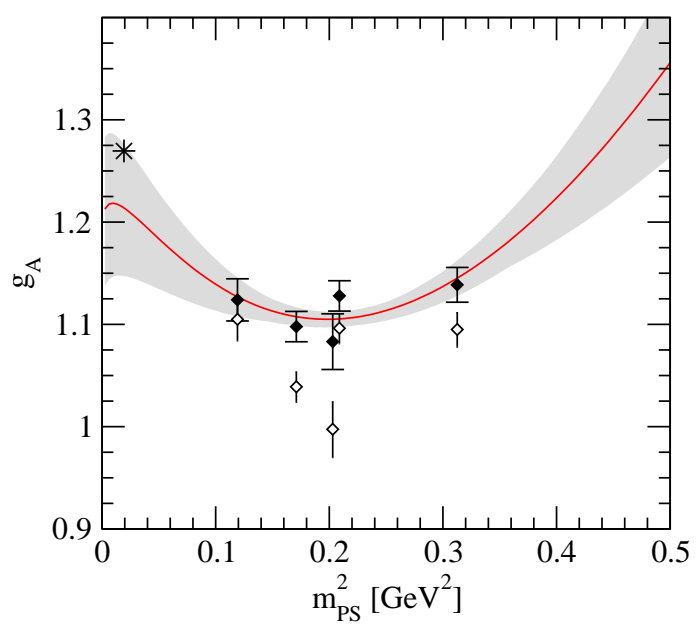

Figure 3: Lattice results for $g_{A}$ together with a fit to an expression obtained from $\mathrm{HB} \chi \mathrm{PT}$. The filled symbols show the lattice results after finite size effects have been corrected.

experimental value, which is however not significant given the large statistical errors. The other fit parameters are of natural size.

\section{Moments of unpolarised structure functions}

Finally, we consider the lowest moment of the unpolarised nucleon structure functions, $\langle x\rangle=$ $A_{2,0}^{q}(0)$, where $A_{2,0}^{q}$ is the first moment of the PDF $H^{q}\left(x, \xi, Q^{2}\right)$ at $\xi=0 .^{2}$ This moment is determined from the matrix element

$$
\left\langle N(\vec{p})\left|\left[\bar{u} \gamma^{\left\{\mu_{1}\right.} \stackrel{\leftrightarrow}{D}^{\left.\mu_{2}\right\}} u\right]\right| N(\vec{p})\right\rangle=2 A_{2,0}^{q}\left[p^{\mu_{1}} p^{\mu_{2}}\right] .
$$

For $O(a)$-improvement of the operator we use the perturbative results obtained in [14], where we inserted the boosted coupling constant $\left(g^{*}\right)^{2}=g^{2} / u_{0}^{4}$. The unknown improvement coefficients are set to zero as the corresponding improvement operators turn out to be small. The renormalisation is both scale and scheme dependent. We have calculated the renormalisation constant $\Delta Z_{\mathrm{v} 2 \mathrm{~b}}^{\text {lat }}(a)$, which translates our lattice results into RGI, non-perturbatively using the RI'-MOM method. For comparison with other results we have to convert our numbers to $\overline{\mathrm{MS}}$. The corresponding factor $\left(\Delta Z_{\mathscr{O}}^{\overline{\mathrm{MS}}}(\mu=2 \mathrm{GeV})\right)^{-1}$ is calculated perturbatively using $\Lambda^{\overline{\mathrm{MS}}}=261(17)(26) \mathrm{MeV}$ [13].

The lattice results for $\langle x\rangle$ in the iso-vector case have been found to be significantly larger than the experimental value. It had been suggested that this quantity may become much smaller at very light quark masses [15]. This has been confirmed by recent calculations in the framework of baryon chiral perturbation theory $(\mathrm{B} \chi \mathrm{PT})[8]$.

We compare our lattice results with these calculations both in the iso-vector case $(u-d)$ and the iso-scalar case $(u+d)$. Note that the latter might be affected by contributions from disconnected terms which we have not calculated so far. In our fits we use phenomenological input for the pion decay constant $F_{0}$, the nucleon mass $M_{0}$ and the nucleon axial coupling $g_{A, 0}$ as listed in Table 1 .

\footnotetext{
${ }^{2}$ Results for $A_{2,0}^{q}$ at $Q^{2}>0$ have been presented by M. Ohtani at this conference [12].
} 

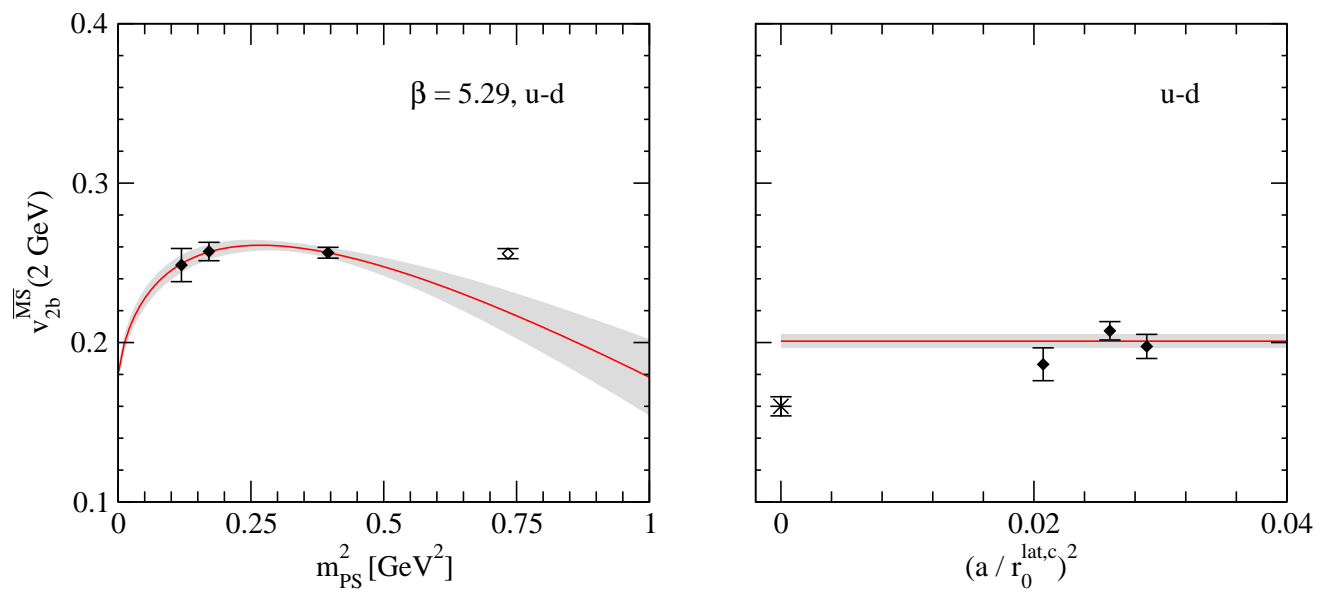

Figure 4: The left plot shows our results for $\langle x\rangle^{(u-d)}\left(m_{\mathrm{PS}}\right)$ at $\beta=5.29$ (the results for other values of $\beta$ are similar). The solid line denotes a fit to an ansatz obtained from $\mathrm{B} \chi \mathrm{PT}$ [8]. The right plot shows $\langle x\rangle^{(u-d)}\left(m_{\mathrm{PS}}=m_{\pi}\right)$ as a function of the lattice spacing together with a fit to a constant.

As suggested in [8] we assume the coupling $\Delta a_{2,0}^{(u-d)} \simeq\langle\Delta x\rangle^{(u-d)}$. We finally end up with two free fit parameters, i.e. $a_{2,0}^{u-d}$ and $c_{8}(\lambda=1 \mathrm{GeV})$ as well as $\left(a_{2,0}^{u+d}\right)$ and $c_{9}(\lambda=1 \mathrm{GeV})$ in the iso-vector and iso-scalar case, respectively. With the statistical errors and the number of fit parameters being sufficiently small we fit the data for different values of $\beta$ separately restricting the fit range to $0<m_{\mathrm{PS}} \lesssim 650 \mathrm{MeV}$ (see Fig. 4 and 5).

In the iso-vector case we still find little indication for $\langle x\rangle^{(u-d)}\left(m_{\mathrm{PS}}\right)$ becoming smaller for $m_{\mathrm{PS}} \rightarrow m_{\pi}$. Currently this is not in contradiction to the $\mathrm{B} \chi \mathrm{PT}$ results since quark masses may still be too large. Furthermore, results at light quark masses may be affected by finite size effects. Within statistical errors the discretisation effects seem to be small. In the iso-scalar case we find evidence for $\langle x\rangle^{(u+d)}\left(m_{\mathrm{PS}}\right)$ to become smaller at lighter quark masses. Our results for $m_{\mathrm{PS}}=m_{\pi}$ are close to the phenomenological value.

\section{Conclusions}

In this paper we presented recent progress in improving our control on the systematic errors for various quantities in the baryon sector. Finite size effects were found to be small in the case of the nucleon mass $m_{\mathrm{N}}$ and large in the case of the nucleon axial coupling $g_{A}$. Investigation of such effects for the delta mass $m_{\Delta}$ and the lowest moment of the unpolarised nucleon structure functions $\langle x\rangle$ is still pending. For all quantities considered here discretisation effects seem to be small, although it remains difficult to distinguish potential $O\left(a^{2}\right)$ effects from other systematic errors. The largest uncertainties stem from the extrapolation of our results to the physical quark masses. We utilised results obtained in $\chi \mathrm{PT}$ to perform these extrapolations. Although most of our lattice simulations have been performed at quark masses outside the region where $\chi \mathrm{PT}$ is expected to converge, our fit parameters turned out to be of natural size and consistent with what is expected from phenomenology.

To get better control on the quark mass dependence, simulations at significantly lighter quark mass are mandatory. Fortunately, with recent algorithmic improvements and even faster computers becoming available exploring the quark mass region with $m_{\mathrm{PS}} \lesssim 300 \mathrm{MeV}$ starts to become feasible. 

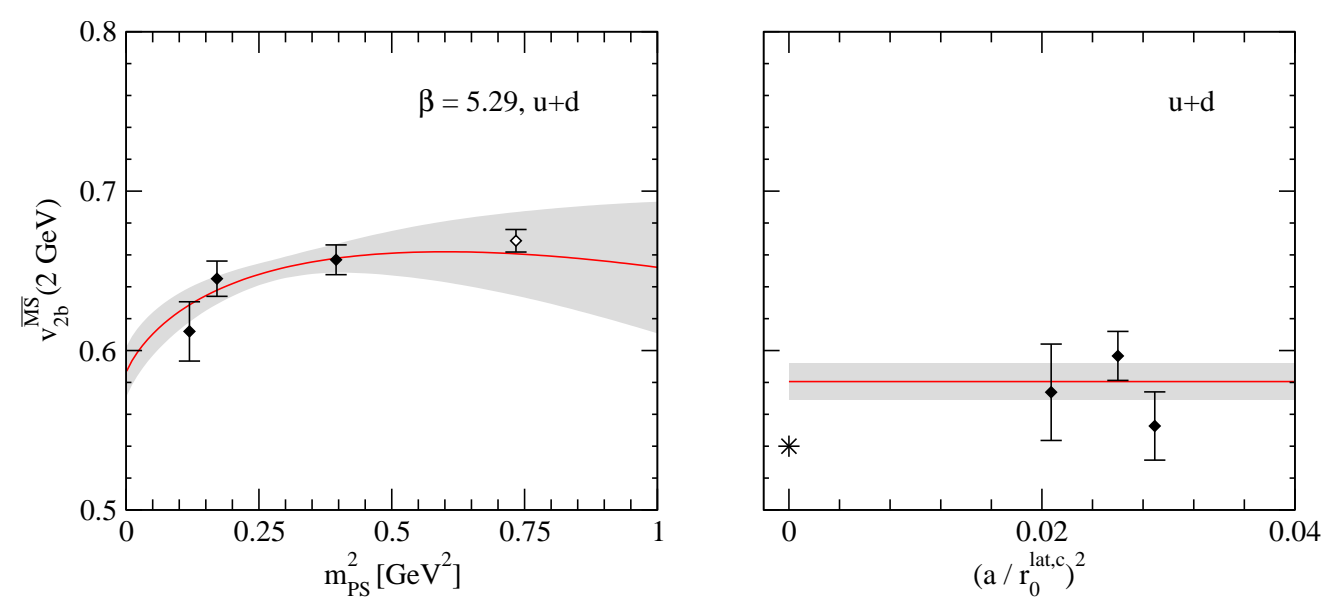

Figure 5: Same as Fig. 4 but for the iso-scalar case $\langle x\rangle^{(u+d)}$.

\section{Acknowledgements}

The numerical calculations have been performed on the Hitachi SR8000 at LRZ (Munich), the Cray T3E at EPCC (Edinburgh) [16] the APE1000 and apeNEXT at NIC/DESY (Zeuthen), the BlueGene/L at NIC/FZJ (Jülich) and EPCC (Edinburgh). Some of the configurations at the small pion mass have been generated on the BlueGene/L at KEK by the Kanazawa group as part of the DIK research programme. This work was supported in part by the DFG, by the EU Integrated Infrastructure Initiative Hadron Physics (I3HP) under contract number RII3-CT-2004-506078.

\section{References}

[1] G. Schierholz et al. [QCDSF Collaboration], PoS (LATTICE 2007) 133.

[2] A. Ali Khan et al. [QCDSF-UKQCD Collaboration], Nucl. Phys. B 689 (2004) 175.

[3] A. Ali Khan et al. [QCDSF Collaboration], Phys. Rev. D 74 (2006) 094508.

[4] M. Göckeler et al. [QCDSF Collaboration], Phys. Rev. D 71 (2005) 034508; W. Schoers et al. [QCDSF Collaboration], POS (LATTICE 2007) 161.

[5] T. A. Gail and T. R. Hemmert, Eur. Phys. J. A 28 (2006) 91.

[6] S. Eidelman et al. [Particle Data Group], Phys. Lett. B 592 (2004) 1.

[7] G. Colangelo and S. Dürr, Eur. Phys. J. C 33 (2004) 543.

[8] M. Dorati, T. A. Gail and T. R. Hemmert, arXiv: nucl-th/0703073.

[9] T. R. Hemmert, M. Procura and W. Weise, Phys. Rev. D 68 (2003) 075009.

[10] V. Bernard, T. R. Hemmert and U. G. Meissner, Phys. Lett. B 622 (2005) 141.

[11] V. Bernard, arXiv:0706.0312 (hep-ph).

[12] M. Ohtani et al. [QCDSF Collaboration], PoS (LATTICE 2007) 158.

[13] M. Göckeler et al. [QCDSF Collaboration], Phys. Rev. D 73 (2006) 014513.

[14] S. Capitani et al. [QCDSF Collaboration], Nucl. Phys. B 593 (2001) 183.

[15] W. Detmold et al., Phys. Rev. Lett. 87 (2001) 172001.

[16] C. R. Allton et al., Phys. Rev. D 65 (2002) 054502. 